\title{
Endovascular therapy for acute tumour-related obstruction of the superior vena cava using a self-expanding Nitinol stent
}

\author{
P Kamusella, Dr Med; C Wissgott, PD, Dr Med; R Andresen, Dr Med
}

Institute of Diagnostic and Interventional Radiology/Neuroradiology, Westkuestenklinikum - Academic Teaching Hospital of the Universities of Kiel, Lübeck and Hamburg, Heide, Germany

Corresponding author: P Kamusella (pkamusella@wkk-hei.de)

\begin{abstract}
Objective. To evaluate, in a retrospective study, the clinical efficacy and safety of the self-expanding Nitinol stent in the superior vena cava to alleviate upper venous congestion.

Method. In 22 patients (15 men, 7 women), a tumour-related compression of the superior vena cava was diagnosed by spiral CT after intravenous application of contrast medium. Clinically, acute superior vena cava syndrome was found in all patients. Histologically, a bronchial carcinoma was present in 14/22, a lymphoma in 6/22, and mediastinal lymphnode metastases ( 1 breast carcinoma, 1 malignant melanoma) in $2 / 22$. After a transfemoral approach, cavography was initially performed. The degree of stenosis was classified according to the Stanford classification. In accordance with the degree of stenosis, a self-expanding Nitinol stent was placed.

Results. Endovascular stent implantation was conducted without complications in all patients. A marked improvement in acute symptoms was observed clinically within 24 hours in all patients. In the follow-up period of up to 2 years, there were no cases of stent migration. In 7/22 patients, the CT follow-ups revealed tumour progression (3/7 after 3 months, 2/7 after 6 months, and 2/7 after 12 months) with evidence of residual stenosis caused by tumour growth through the stent mesh. During the follow-up period, 15/22 patients died (mean survival 6.4 months).

Conclusion. Self-expanding Nitinol stents provide endovascular therapy for superior vena cava syndrome, having a high radial expansive force and the facility to be placed precisely, and alleviating acute, life-threatening symptoms in the palliative situation.
\end{abstract}

S Afr J Rad 2013;17(4):123-127. DOI:10.7196/SAJR.864

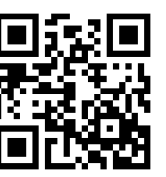

Superior vena cava syndrome (SVCS) is characterised by venous congestion and increased pressure resulting from displacement or compression of the superior vena cava (SVC). Venous congestion leads to swelling of the soft tissues of the face and neck, dyspnoea and dysphagia, extending to glottal and laryngeal oedema and the development of cerebral oedema with headache and clouded consciousness. The evolving life-threatening situation generally takes a slow and progressive course, and is rarely acute. ${ }^{[1]}$

The cause of the obstruction can be a thrombosis or an invasion or external compression of the SVC by topographically adjacent pathological processes, especially originating in the right lung, lymph nodes and other mediastinal structures. In rare cases, a combination of external compression and cava thrombosis is present. ${ }^{[2]}$ In $74-95 \%$ of cases, malignant tumours are the primary cause of upper venous congestion, which typically results from an advanced lung carcinoma (about $80 \%$ ), more rarely from a lymphoma (about 15\%) or metastasis of an extrathoracic tumour (about 5\%). Up to $5 \%$ of patients with a lung carcinoma develop upper venous congestion during their illness. ${ }^{[3]}$ As a result of the usually rapidly advancing central tumour growth, small cell lung carcinomas (SCLCs) lead to compression of the SVC more often than do non-small cell lung carcinomas (NSCLCs).
The increasing use of central venous catheters or the implantation of cardiac pacemakers can lead to thromboses or central nervous obstructions and thus also, in rare cases, result in SVCS. ${ }^{[4]}$

In the case of life-threatening SVCS, rapid assistance is required to alleviate the acute symptoms and improve quality of life. Endovascular therapy by stent implantation has proved to be an effective treatment method here. Self-expanding metal stents, such as the Wallstent (Boston Scientific, Nastrick MA, USA) have been used till now. ${ }^{[6]}$

\section{Objective}

This retrospective study (2009-2013) assessed the clinical efficacy and safety of self-expanding Nitinol stents (Sinus XL, Optimed, Ettlingen, Germany) in the treatment of acute, tumour-related SVCS. These stents have been authorised for the treatment of cava obstructions and for vena cava syndrome.

\section{Method}

The indication for intervention was previously established by an interdisciplinary tumour board, consisting of internists/oncologists, surgeons, gynaecologists, neurosurgeons, radio-oncologists and radiologists. Pre-interventionally, patients were advised by the treating 


\begin{tabular}{|c|c|c|c|c|c|c|}
\hline Patient & Sex & Age & Cause of SVC syndrome & Stanford classification & Stenosis lengths $(\mathrm{cm})$ & Survival (months) \\
\hline 1 & M & 77 & Bronchial carcinoma (NSCLC) & Type III & 4.5 & 2 \\
\hline 2 & $\mathrm{~F}$ & 40 & $\begin{array}{l}\text { Lymph node metastases of breast } \\
\text { cancer }\end{array}$ & Type I & 2.6 & alive (24) \\
\hline 3 & M & 40 & Bronchial carcinoma (SCLC) & Type II & 3.8 & 13 \\
\hline 4 & $\mathrm{~F}$ & 75 & Lymphoma & Type III & 5 & 3 \\
\hline 5 & M & 62 & Bronchial carcinoma (NSCLC) & Type II & 3.5 & 7 \\
\hline 6 & M & 75 & Bronchial carcinoma (NSCLC) & Type III & 4.8 & 4 \\
\hline 7 & M & 74 & Lymphoma & Type II & 3.8 & 11 \\
\hline 8 & M & 76 & Lymphoma & Type III & 4 & 4 \\
\hline 9 & M & 44 & Lymph node metastases of melanoma & Type I & 2.8 & alive (5) \\
\hline 10 & $\mathrm{~F}$ & 66 & Bronchial carcinoma (SCLC) & Type II & 3.6 & 5 \\
\hline 11 & M & 69 & Bronchial carcinoma (SCLC) & Type II & 3.4 & 8 \\
\hline 12 & M & 78 & Lymphoma & Type III & 4.7 & 5 \\
\hline 13 & M & 61 & Bronchial carcinoma (NSCLC) & Type I & 2.9 & alive (9) \\
\hline 14 & M & 64 & Lymphoma & Type II & 3.5 & alive (12) \\
\hline 15 & $\mathrm{~F}$ & 65 & Bronchial carcinoma (SCLC) & Type II & 3.5 & 7 \\
\hline 16 & $\mathrm{~F}$ & 71 & Bronchial carcinoma (SCLC) & Type II & 3.5 & 8 \\
\hline 17 & M & 49 & Bronchial carcinoma (SCLC) & Type II & 3.8 & 6 \\
\hline 18 & M & 55 & Bronchial carcinoma (NSCLC) & Type I & 2.7 & alive (3) \\
\hline 19 & M & 72 & Bronchial carcinoma (SCLC) & Type III & 4.8 & alive (4) \\
\hline 20 & $\mathrm{~F}$ & 77 & Bronchial carcinoma (SCLC) & Type II & 3.9 & alive (8) \\
\hline 21 & M & 80 & Bronchial carcinoma (SCLC) & Type II & 3.5 & 9 \\
\hline 22 & $\mathrm{~F}$ & 79 & Lymphoma & Type III & 5 & 4 \\
\hline
\end{tabular}

interventionalist about the course of the procedure, risks and expected outcome. Written consent was obtained from all patients before the intervention was performed.

\section{Patients}

In 22 patients ( 15 men, 7 women, average age 65.9 years), a tumourrelated compression of the SVC was diagnosed by multidetector computed tomography (MDCT) of the chest (Table 1). After injecting $100 \mathrm{ml}$ Imeron 350 via an antecubital vein in the left arm at a rate of $3 \mathrm{ml} / \mathrm{s}$ and a delay of $30 \mathrm{~s}$, CT was performed, with a $3 \mathrm{~mm}$ slice thickness and $3 \mathrm{~mm}$ reconstruction interval. Clinically, acute SVCS was found in all patients.

Acute or rapidly progressive dyspnoea and a swelling of the soft tissues of the face and neck led to inpatient admission in all cases $(22 / 22)$. All patients were also characterised by dilatation of the jugular veins. Dilated veins of the chest wall were also found in 7/22 patients. Histologically, 14/22 had a lung carcinoma (9 SCLC, 5 NSCLC), 6/22 a lymphoma (non-Hodgkin lymphoma) and 2/22 mediastinal lymph node metastases ( 1 breast carcinoma, 1 malignant melanoma).

At the time of endovascular therapy, a definitive histological diagnosis was known in 18/22 patients. A CT-assisted biopsy was conducted in $4 / 22$ over the further course. If the tumour entity was known, intensive therapy (radiochemotherapy) was started or continued. In planning the procedure, the chest CT was evaluated to determine the extent and cause of the occlusion, and thrombotic substrate was searched for. In addition, cavography was performed immediately before stent implantation for an exact assessment of the venous system.

\section{Procedure}

Under sterile conditions, after local anaesthesia with Mecain 2\%, the Seldinger technique was used to puncture the right common femoral vein just below the inguinal ligament. A 10-French sheath (Terumo, Tokyo, Japan) was then inserted, followed by wire probing (Terumo Stiff guide wire 0.035 inch) of the SVC and insertion of a 4-French pigtail catheter. To prepare serial angiograms in different planes, an automated contrast medium application system $(10-15 \mathrm{ml} / \mathrm{s}$, total of $20-25 \mathrm{ml}$ per series) was used for the cavography. Examinations were conducted on a digital flat detector angiography system (AlluraXper FD 20, Philips Healthcare, Best, the Netherlands) by means of pulsed fluoroscopy (image frequency 15/s).

After cavography (Fig. 1), the stenosis was classified according to the Stanford classification ${ }^{[7]}$ system into 4 types: $(i)$ type I describes a partial obstruction of the SVC (up to 90\%) with remaining patency and antegrade perfusion of the azygos vein; (ii) type II shows increasing obstruction (90 - 100\%); (iii) type III is complete obstruction with reverse circulation in the azygos vein; and (iv) type IV is characterised by complete obstruction of the SVC and the azygos vein, with the development of collateral circulations via the chest wall and the internal mammary veins. 


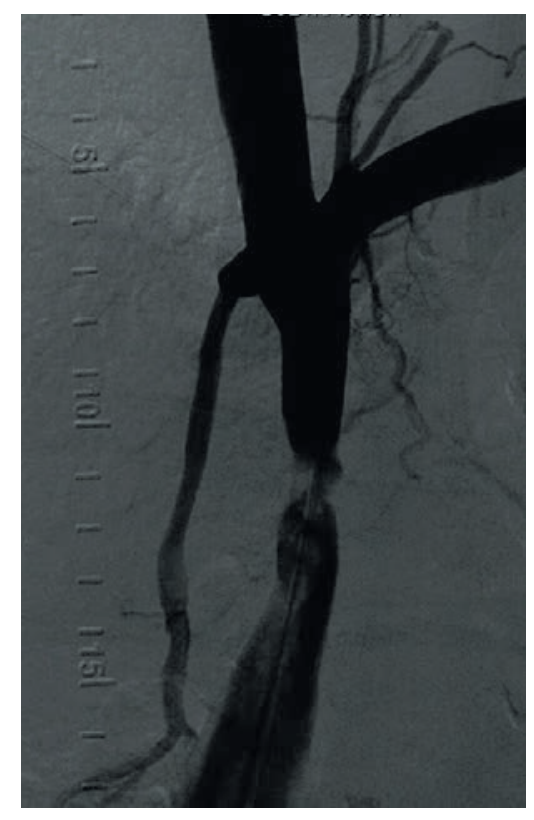

Fig. 1. Digital subtraction angiography of the superior vena cava (cavography) with evidence of severe lumen reduction (type II, Stanford classification).

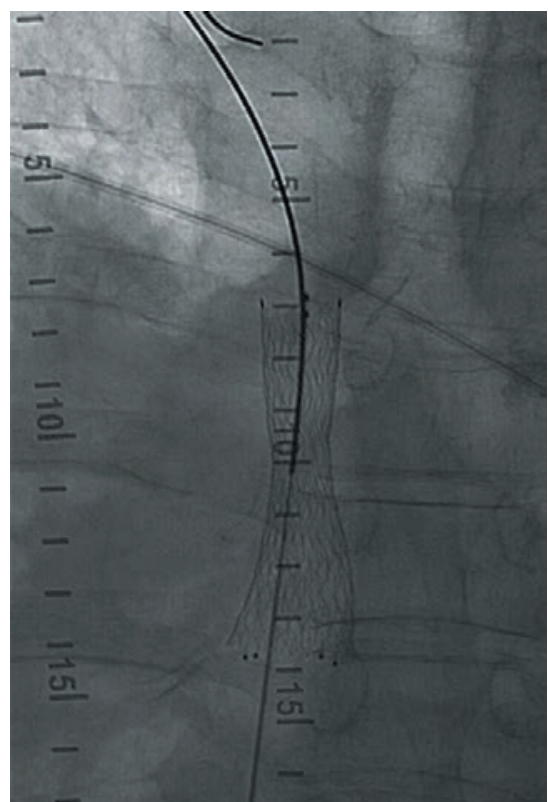

Fig. 2. Superior vena cava at the time of stent deployment.

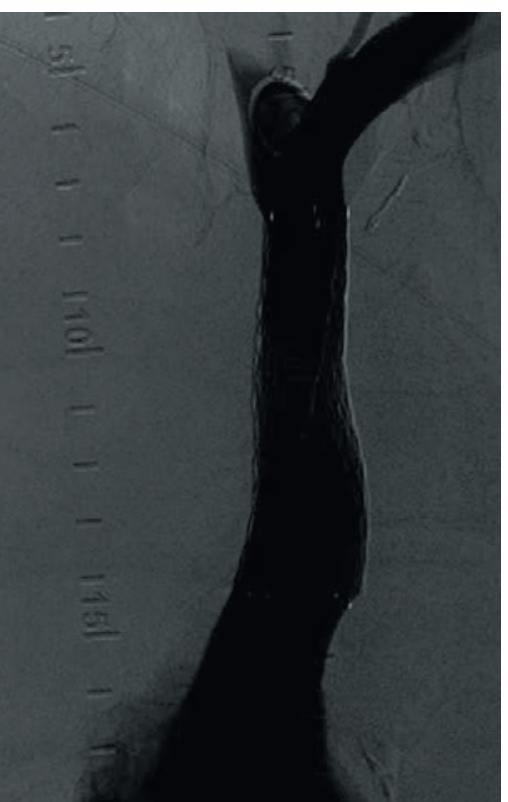

Fig. 3. Cavography after stent implantation and balloon angioplasty. Presentation of the stented uncaged superior vena cava with correct stent position and a regression of collateral circulation.
Corresponding to the stenosis, a selfexpanding Nitinol stent was placed. Despite the high radial expansion force of the stent, residual tumour-related constrictions were subsequently dilated with a balloon (Zelos, Optimed, Ettlingen, Germany) in all patients for optimisation in the area of the stenosis. A non-compliant balloon was used, which was chosen to be slightly smaller in relation to the stent diameter and with a length of $40 \mathrm{~mm}$. A stent with a diameter of $20 \mathrm{~mm}$ was subsequently dilated with the aid of a balloon with a diameter of $18 \mathrm{~mm}$, without exceeding the working pressure of 4 bar. Accordingly, a $20 \mathrm{~mm}$ balloon was used for a $22 \mathrm{~mm}$ stent. The stent length and the diameter were determined on the basis of the $\mathrm{CT}$ and cavography. The stent length was chosen according to the stenosis. The stent diameter was determined on the basis of the diameter of the SVC in the normal calibre range, taking into account respiratory variability (Fig. 2).

Before stent implantation, each patient received an intravenous bolus of $5000 \mathrm{IU}$ heparin. Serial angiograms in 2 planes (AP and $45^{\circ}$ oblique projections) were then prepared to check for adequate recanalisation of the SVC. The treatment was considered to have been completed successfully if the stent was in the correct position and showed a normal contrast medium outflow in the cavogram, and the collaterals had markedly receded (Fig. 3). After removal of the sheath from the common femoral vein, the puncture site was manually compressed until haemostasis was achieved, and a light compression bandage was applied for at least $6 \mathrm{~h}$. Bed rest was recommended for at least $12 \mathrm{~h}$. On the following day, a renewed chest CT was conducted to check the postinterventional outcome.

The follow-up period included presentation in the oncological outpatient department with a clinical examination and radiological diagnostics (chest CT). The follow-ups were after 3 months up to 24 months, and were carried out in those patients who had survived the course. In the event of renewed clinical deterioration, the algorithm was departed from. Restenosis was defined as $>50 \%$ lumen reduction. The indication for re-intervention was discussed by an interdisciplinary team only if clinical symptoms recurred.

\section{Results}

The cavography performed before stent placement revealed a partial obstruction of the SVC in $4 / 22$ (18.2\%, type I) and in $11 / 22$ (50\%, type II) of the patients. A complete obstruction of the SVC (type III) was seen in $7 / 22(31.8 \%)$ of the patients. No cases of complete obstruction of the SVC and the azygos vein (type IV) were detected.
Additional cava thrombosis was not found. Stenosis lengths averaging $3.8 \mathrm{~cm}(2.5-5 \mathrm{~cm})$ were measured. Stents with a length of $60 \mathrm{~mm}$ were used in all patients, with sufficient cover of the stenosis. A stent with a diameter of $20 \mathrm{~mm}$ was chosen for 2 patients. In 20 patients, stents of $22 \mathrm{~mm}$ diameter were used.

A self-expanding Nitinol stent was implanted in all cases. The technical success rate was $100 \%$, with optimal cover of the stenosis. In all $(22 / 22)$ cases, the stenosis was successfully bridged with a single stent. There were no stent-related complications such as stent migration. Complications relating to the procedure, especially in the area of the stenosis or the puncture site, were not observed. In the follow-up period of up to 2 years, no stent migration was found. An improvement in clinical symptoms was observed within $24 \mathrm{~h}$ after stent implantation in all cases. Of subjective importance for the patients was the improvement in dyspnoea, which meant that they could assume a lying position without difficulty, and oxygen substitution could be discontinued. Objective signs of improvement were the decline in facial and neck oedema and regression of the venous cervical and thoracic dilatation.

Patients with known tumour disease continued their intensive therapy (radiochemotherapy). Patients with newly discovered 
tumour disease (3 SCLC and 1 non-Hodgkin lymphoma) were able to start oncological treatment shortly after histological clarification.

In the follow-up CT, tumour progression was found in $7 / 22$ patients (3/7 after 3 months, 2/7 after 6 months, and 2/7 after 12 months), with evidence of restenosis caused by tumour growth through the stent mesh (Fig. 4). This resulted in a renewed SVCS. CT detected a restenosis $<50 \%$ in $4 / 7$ and a stenosis $>50 \%$ in $3 / 7$ of the patients. For the primary patency rate, a value of $86.4 \%$ was obtained. During the follow-up period, $15 / 22$ (68.2\%) of the patients died, yielding a mean survival of 6.4 months. Only one patient has so far survived a period of 2 years after stent implantation, and continues to be followed along with the remaining patients.

\section{Discussion}

Rapid diagnostics and effective therapy are necessary to correct tumourrelated compression of the SVC within the context of acute life-limiting symptoms.

\section{Imaging}

In conventional projection radiography of the chest, the majority of patients with SVCS show pathologies that present mainly as a mediastinal broadening, and to a lesser extent also with accompanying pleural effusions.

MDCT is the method of choice; it clarifies the extent of venous obstruction, identifies the venous collateral system and enables an estimation of the cause of the SVCS. In practice, obstruction of the SVC is often diagnosed on routine contrast-enhanced chest CT via an antecubital vein in the left arm. ${ }^{[8]}$ To avoid lack of opacification or streak artefacts, Qanadli et al. ${ }^{[9]}$ recommend a simultaneous bilateral antecubital vein injection; Sheth et al. ${ }^{[8]}$ prefer a delay of $60 \mathrm{~s}$, instead of $30 \mathrm{~s}$. In the literature, typically $120 \mathrm{ml}$ (up to $180 \mathrm{ml}$ ) of contrast medium was injected. ${ }^{[8,9]}$ Our experience with a unilateral injection of $100 \mathrm{ml}$ contrast medium and a delay of $30 \mathrm{~s}$ has been good.

In our study, CT also served as a basis for further therapy planning within the context of an interdisciplinary therapeutic decision. MRI is another method that can be used to image the central venous system, supplying valuable information for therapy planning, ${ }^{[10]}$ but this was not conducted in our patient population.

Based on the CT findings, conventional imaging of the veins was performed within the context of the procedure for stent implantation in our study. Cavography enables assessment of the obstruction and visualisation of the collateral circulations. ${ }^{[7]}$ Partial obstructions (types I and II; up to $68 \%$ ) and complete obstruction (type III; 32\%) of the SVC were detected most frequently in our study. Complete obstructions including the azygos vein were not found. In their patient population with 27 venographies performed, Stanford et al..$^{[7]}$ most often described type IV obstructions (37\%), followed by type II and III (26\% each), while type I (11\%) occurred less frequently. Lanciego et al. ${ }^{[11]}$ showed a similar distribution of the obstruction types (type I: $9 \%$; type II: $40 \%$; type III: $28 \%$; type IV: $23 \%$ ).

\section{Therapy}

Various therapeutic options are available, with the aim of rapidly alleviating the acute symptoms and improving the quality of the remaining time available to the patient in the palliative situation. General and pharmacotherapeutic treatments (e.g. bed rest and raised upper body, diuretics, cortisone anticoagulation) can alleviate symptoms, but only show limited clinical success. Standard tumour treatment procedures are radiotherapy and chemotherapy, or a combination of the two, which create relief by reducing the tumour mass. An improvement in symptoms is achieved in $80 \%$ of patients with these treatment methods. In the case of NSCLC, a decline in symptoms of $46-63 \%$ is seen with radiotherapy, and of $59 \%$ with chemotherapy. In the case of SCLC, success rates of $62-80 \%$ with chemotherapy and $83 \%$ with combined radiochemotherapy have been achieved. ${ }^{[3]}$ However, these therapeutic options take 7 - 14 days before symptoms improve, and in some studies up to 4 weeks. Aggressive radiotherapy (8Gy 3 times a week for 3 weeks) achieved a response of up to $90 \%$, but this treatment also leads to an increase in radiation-related complications, such as tumour necrosis with fever attacks, the risk of bleeding and even perforation of the SVC. In addition, side-effects such as nausea and vomiting, skin irritations and oesophagitis have been observed.

In the light of morbidity, mortality and limited life expectancy, surgical resection with vascular reconstruction is performed in only the rarest of cases in this patient population with acute symptoms. In contrast, surgical interventions are effective in benign causes of upper venous congestion and show relatively few complications. If, in the case of a malignant cause of upper venous congestion, a mean life expectancy of 6 months is assumed, which is analogous to the 6.4 months observed in our patient population, the remaining time should comprise alleviating the symptoms and maintaining quality of life.

\section{Endovascular stent implantation}

In the event of severe symptoms, endovascular stent implantation represents the method of choice for acute treatment and is characterised by its capability of providing rapid relief from the acute symptoms (within $24 \mathrm{~h}$ ) in up to $90 \%$ of cases. The technical success rate is around $95-100 \%$. In our patient population, an improvement in dyspnoea and facial swelling in particular, was observed within $24 \mathrm{~h}$, with a technical success rate of $100 \%$.

The transfemoral approach proved effective for placing the stent. The right common femoral vein is preferred, while other venous approaches such as the internal jugular vein or the peripheral veins of the upper arm are used in special cases only. ${ }^{[6]}$ Based on this experience, we also used a rightsided transfemoral approach, after previous skin disinfection and local anaesthesia, in all cases in our study. In none of the cases was it necessary to puncture a different vein region. When selecting the stent size, the stent length should exceed the measured obstruction cranially and caudally by $1 \mathrm{~cm}$ each way, while the stent diameter should be adapted to the SVC in its unobstructed state. ${ }^{[6]}$ Regarding efficacy, no significant difference is found in the literature between the 3 most commonly used stents (Gianturco Z-stent, Cook Medical Inc., Bloomington, USA; Palmaz stent, Johnson \& Johnson, Warren, USA; Wallstent, Boston Scientific, Natrick, USA). In the past, the Gianturco Z-stent has been described as having an increased rate of stent migration and stent malpositioning. These stents are self-expanding and characterised by largely resistance-free stenosis passage and a wide range of lengths and widths. Compared with the standard metal stents, more recent self-expanding stents made of a nickeltitanium alloy (Nitinol) have a higher precision in stent placement within the stenosis, an only minimal stent shortening, lower thrombogenicity and a higher radial expansion force, while remaining highly flexible. ${ }^{[12,13]}$ In our study, a 10-F Nitinol stent system authorised for SVCS was used and is available in various widths $(16-34 \mathrm{~mm})$ and lengths $(30-100 \mathrm{~mm})$. At stenosis lengths of $2.5-5 \mathrm{~cm}, 6 \mathrm{~cm}$ stents were applied with a diameter of 2.0 and $2.2 \mathrm{~cm}$. Overlapping stent implantations were not necessary in 


\section{ORIGINAL ARTICLE}
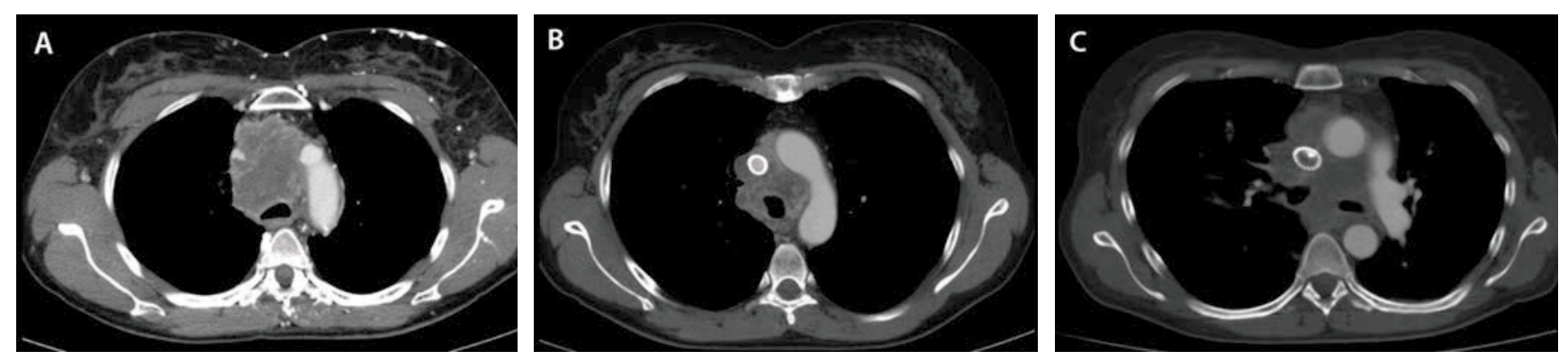

Fig. 4. (A) A central, small cell lung cancer in the upper mediastinum with encasement of mediastinal structures, in particular the superior vena cava. In the area of the chest wall, especially on the left side, an increased venous drawing is visible at skin level. (B) Follow-up computed tomography (at 3 months) after stent implantation. The stent in the superior vena cava is in the correct position and the vascular lumen is free perfused. (C) Follow-up computed tomography (at 6 months) after stent implantation. Restenosis of the superior vena cava by progression of tumour growth, in particular through the stent mesh. A planned re-intervention was not performed because the patient died from the consequences of the disease.

our study, as the available stent sizes enabled complete coverage of the obstruction with one stent in each case.

If both brachiocephalic veins are involved in the obstruction, the patients may benefit from a unilateral stent implantation. Unilateral treatment is sufficient; on the one hand, blood flow is also possible from the contralateral side through the stent mesh; on the other, blood circulation takes place via cervicothoracic collateral channels. ${ }^{[6]}$ Bilateral recanalisation does not improve the outcome. In the case of parallel positioning of the stents in the SVC, the stents may not expand fully, which may be accompanied by increased recurrence of SVCS. ${ }^{[11,14]}$

If a central venous catheter is in place, various options present. If chemotherapy has been concluded, removal of the catheter may be considered prior to stent placement. If it is still required, an attempt can be made to move the catheter tip temporarily into another position before implantation of the stent, e.g. into the cervical or brachial veins, and move it back afterwards. If this is not possible, the central venous catheter can be overstented. ${ }^{[6]}$ Balloon dilatation may be necessary before stent implantation. Though desirable in cases of benign SVC obstruction, it is not always required in malignant stenoses, but may be necessary for total obstructions. ${ }^{[6]}$ After stent implantation, balloon dilatation is recommended to optimise an eccentric stent position and remove residual stenoses. If a thrombus is additionally present, it can be pushed back further by angioplasty in the case of incomplete stent expansion. ${ }^{[6]}$ So-called noncompliant balloons were exclusively used in our study; they are characterised by the fact that they only change their diameter minimally upon dilatational pressure and therefore minimise the risk of overexpansion. Complications of stent placement are seen in $3-7 \%$ of patients. ${ }^{[1]}$ The early complications include pulmonary artery embolism, stent migrations, haematomas and bleeding, ranging up to the rare perforation or rupture of the SVC. ${ }^{[15]}$ No such complications were observed in our study.

Failures after stent implantation may be linked to tumour invasion into the SVC through the stent mesh and thrombus formation. Lanciego et al. ${ }^{[11]}$ described such re-occlusion as a result of tumour growth in $16 / 149$ patients $(\sim 10 \%)$ and in only 2 cases as a result of a thrombus in their patient population. In our study, tumourrelated restenoses were seen in about 30\% of patients over a period up to 12 months, the high tumour burden and deterioration of the overall situation meaning that renewed intervention was no longer indicated. If a relevant stenosis is defined as a lumen constriction $>50 \%$, 3 restenoses were present in our patient population, thus yielding a primary patency rate of $86.4 \%$. This value is slightly higher than that achieved by Da Ines et al., ${ }^{[6]}$ using Wallstents, of about $81 \%$.

In the case of re-obstruction, re-intervention should fundamentally be discussed. ${ }^{[3,11]}$ In such cases, the use of covered stents is conceivable, but possible thrombogenicity and potential risk of blocking afferent vessels must be borne in mind. ${ }^{[6]}$ At present, only individual case reports are available ${ }^{[16]}$ but they demonstrate that tumour growth through the stent mesh, or stent migration with vascular rupture, can be treated with a covered stent. Covered stents were not used in our study.

Within the context of treatment of SVCS, clear recommendations are not available for anticoagulation and platelet aggregation inhibition. While most interventionalists routinely use a heparin bolus of $5000 \mathrm{IU},{ }^{[6]}$ opinion is divided about follow-up treatment and its duration. For example, the interdisciplinary S3 guideline of the German Respiratory Society and the German Cancer Society says that anticoagulation is generally not necessary. Therefore, after stent implantation, a middle way must be found between the risk of early re-occlusion and haemorrhagic complications. To avoid thrombus formation, platelet aggregation inhibition with acetylsalicylic acid and/ or clopidogrel is to be recommended. If a thrombus is present, anticoagulation with heparin is necessary for the duration of tumour treatment. ${ }^{[6]}$ The duration of anticoagulation is dependent on therapyrelated factors, and side-effects and should be discussed in conjunction with attending oncologists on an individual basis.

\section{Conclusion}

Self-expanding Nitinol stents provide endovascular therapy for SVCS with a tool that can be placed precisely and that alleviates the patient's acute, life-threatening symptoms quickly and effectively in the palliative setting. As a result of post-interventional clinical improvement, it is then possible to pass on the patient for further targeted oncological therapy. 